\title{
UICN
}

\section{Lignes directrices de prise en charge des organismes vivants confisqués}

Neil Maddison, éditeur

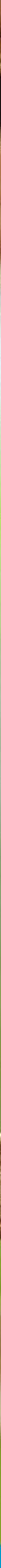




\section{À propos de I'UICN}

L'UICN est une union de Membres composée de gouvernements et d'organisations de la société civile. Elle offre aux organisations publiques, privées et non-gouvernementales les connaissances et les outils nécessaires pour que le progrès humain, le développement économique et la conservation de la nature se réalisent en harmonie.

Créée en 1948, l'UICN s'est agrandie au fil des ans pour devenir le réseau environnemental le plus important et le plus diversifié au monde. Elle compte avec l'expérience, les ressources et le poids de ses plus de 1300 organisations Membres et les compétences de ses plus de 13000 experts. Elle est l'un des principaux fournisseurs de données, d'évaluations et d'analyses sur la conservation. Sa taille lui permet de jouer le rôle d'incubateur et de référentiel fiable de bonnes pratiques, d'outils et de normes internationales.

L'UICN offre un espace neutre où diverses parties prenantes - gouvernements, ONG, scientifiques, entreprises, communautés locales, groupes de populations autochtones, organisations caritatives et autres - peuvent travailler ensemble pour élaborer et mettre en œuvre des solutions pour lutter contre les défis environnementaux et obtenir un développement durable.

Travaillant de concert avec de nombreux partenaires et soutiens, l'UICN met en œuvre un portefeuille vaste et divers de projets liés à la conservation dans le monde. Associant les connaissances scientifiques les plus pointues et le savoir traditionnel des communautés locales, ces projets visent à mettre un terme à la disparition des habitats, à restaurer les écosystèmes et à améliorer le bien-être des populations.

www.iucn.org/fr

https://twitter.com/IUCN/

\section{Commission de sauvegarde des espèces (CSE) de I'UICN}

Avec ses plus de 8000 membres, la Commission de sauvegarde des espèces (CSE) est la plus grande des six commissions expertes de l'UICN. Elle lui permet d'influencer, d'encourager et d'aider les sociétés à préserver la biodiversité en les sensibilisant au statut des espèces et aux menaces qu'elles affrontent, en leur prodiguant des conseils, en élaborant des politiques et des directives, en facilitant la planification des projets de conservation et en catalysant les actions de conservation.

Les membres de la CSE appartiennent à l'un ou plusieurs des 140 groupes de spécialistes, autorités de liste rouge et groupes de travail, qui se concentrent sur un groupe taxonomique (plantes, champignons, mammifères, oiseaux, reptiles, amphibiens, poissons et invertébrés) ou sur un enjeu propre à une discipline particulière, tel que l'exploitation durable des ressources naturelles et les moyens de subsistance, la réintroduction des espèces, la santé de la faune et la flore, le changement climatique et la planification des projets de conservation.

www.iucn.org/theme/species/about/species-survival-commission 


\section{Lignes directrices de prise en charge des organismes vivants confisqués}

Neil Maddison, éditeur 
La terminologie géographique employée dans cet ouvrage, de même que sa présentation, ne sont en aucune manière l'expression d'une opinion quelconque de la part de l'UICN sur le statut juridique ou l'autorité de quelque pays, territoire ou région que ce soit, ou sur la délimitation de ses frontières. Les opinions exprimées dans cette publication ne reflètent pas nécessairement celles de l'UICN.

L'UICN rejette toute responsabilité en cas d'erreurs ou d'omissions intervenues lors de la traduction en français de ce document dont la version originale est en anglais. En cas de divergences, veuillez vous référer à l'édition originale. Titre de l'édition originale : UICN (2019). Guidelines for the management of confiscated, live organisms. https://doi.org/10.2305/IUCN.CH.2019.03.en

Le présent ouvrage a pu être publié grâce à un soutien financier de l'Environment Agency Abu Dhabi. La traduction de cet ouvrage a été rendue possible grâce au soutien de l'International Fund for Animal Welfare (ifaw).

Publié par

UICN, Gland, Suisse.

\section{Droits d'auteur}

(C) 2019 UICN, Union internationale pour la conservation de la nature et de ses ressources.

La reproduction de cette publication à des fins non commerciales, notamment éducatives, est permise sans autorisation écrite préalable du détenteur des droits d'auteur à condition que la source soit dûment citée.

La reproduction de cette publication à des fins commerciales, notamment en vue de la vente, est interdite sans autorisation écrite préalable du détenteur des droits d'auteur.

\section{Citation}

UICN (2019). Lignes directrices de prise en charge des organismes vivants confisqués. Gland, Suisse: UICN. iv + 40 p.

\section{Éditeur}

Neil Maddison

\section{ISBN}

978-2-8317-1997-9 (PDF)

978-2-8317-1998-6 (empreinte)

DOI

https://doi.org/10.2305/IUCN.CH.2019.03.fr

Traduction

IFAW, International Fund for Animal Welfare

Photo couverture

Jhonathan Miranda

Imprimé par

IFAW, International Fund for Animal Welfare

Disponible auprès de

UICN, Union internationale pour la conservation de la nature

Rue Mauverney 28

1196 Gland

Suisse

www.iucn.org/resources/publications

Cet ouvrage est imprimé sur papier Refutura, $100 \mathrm{gr}$, en papier recyclé FSC, neutre en $\mathrm{CO} 2$ et $100 \%$ vieux.

Cet ouvrage est imprimé sur du papier obtenu à partir de fibre de bois provenant de forêts bien gérées, certifiées selon les normes du Forest Stewardship Council (FSC). 


\section{Sommaire}

Remerciements iv

1 Contexte 1

1.1 Énoncé des besoins $\quad 1$

1.2 Objectif des présentes lignes directrices 3

1.3 Principes de précaution 3

1.4 Approche proactive $\quad 4$

1.5 Définitions $\quad 5$

2 Critères de prise de décision $\quad 7$

2.1 L'échelle individuelle $\quad 7$

2.2 Ressources disponibles $\quad \mathbf{8}$

2.3 Facteurs juridiques et conventions internationales $\quad \mathbf{9}$

3 Planification des interventions 10

3.1 Soins immédiats à court terme $\quad 10$

3.2 Processus de collecte d'informations et d'évaluation initiale $\mathbf{1 1}$

3.2.1. Identification des espèces $\mathbf{1 1}$

3.2.2. Statut de conservation et priorités de I'UICN 12

3.2.3. Santé 12

3.2.4. Possibilités de survie $\quad 13$

3.2.5. Motif de confiscation $\quad 14$

3.2.6. Pays d'origine et d'arrivée $\quad 15$

3.2.7. Espèces envahissantes reconnues $\quad 15$

3.3 Détention provisoire $\quad 16$

3.4 Arbre décisionnel pour la prise en charge à long terme $\quad \mathbf{1 6}$

3.4.1. Rapatriement $\quad 16$

3.4.2. Transfert (ou restitution de l'individu confisqué dans la nature) $\quad 18$

3.4.3. Soins en captivité à long terme $\quad 19$

3.4.4. Mise à mort sans cruauté/destruction 21

4 Conclusion 22

Annexe 1: Réseau consultatif sur la confiscation: mandat 25

Annexe 2: Sources d'informations utiles et liens hypertexte correspondants $\quad 29$

Annexe 3: Collecte d'informations et d'évaluation initiale $\mathbf{3 3}$

Annexe 4: Arbre décisionnel - Espèces confisquées 37 


\section{Remerciements}

Kira Mileham a grandement contribué à la compilation et à l'intégration d'avis et de retours d'information divers. Mark Stanley Price (université d'Oxford) et Tomasina Oldfield (TRAFFIC) ont réalisé des apports très utiles à la présente publication. Les participants à la conférence 2014 de Wild Animal Rescue Network et les membres du groupe de spécialistes de la planification de la conservation de I'UICN (auparavant groupe de spécialistes de l'élevage conservatoire) ont également réalisé des apports importants et l'éditeur les remercie de leur participation. Rachel Hogan (OBE), d'Ape Action Africa, et Valentina Rodrigues, de la force de police environnementale colombienne, ont également participé à la préparation des lignes directrices grâce à leur retour d'information. Nous remercions tout particulièrement feu Maria Boyd pour ses remarques sur la prise en charge des animaux vivants confisqués en Chine. 
Au cours des dix dernières années, le problème du trafic illicite des espèces sauvages est devenu très grave, mais, face à cette menace, les autorités ont répondu à l'appel, redoublant d'efforts pour corriger la situation. Pour mener à bien leur action, elles doivent souvent saisir et confisquer des espèces sauvages, qu'il s'agisse de plantes, d'animaux ou de champignons. Bien que ces confiscations concernent parfois des spécimens non vivants faisant partie d'objets, de produits alimentaires ou de produits médicamenteux, elles touchent souvent des espèces vivantes. Sachant que les confiscations se font de plus en plus fréquentes et portent souvent sur un grand nombre d'individus, il est important d'adopter des approches fondées sur les bonnes pratiques pour prioriser la conservation et assurer la sauvegarde de ces plantes et animaux. Le présent document est destiné à fournir une orientation en la matière.

\section{1 Énoncé des besoins}

L'Union internationale pour la conservation de la nature (UICN) est le fer de lance international de la conservation de la biodiversité, de l'exploitation durable des ressources naturelles, des solutions fondées sur la nature et des moyens de gouvernance correspondants, et aide les autorités compétentes à trouver des solutions réalistes et pratiques aux défis environnementaux. L'UICN produit des « lignes directrices " destinées à orienter les décisions touchant à la gestion des ressources naturelles. Les premières lignes directrices de l'UICN relatives à l'utilisation des animaux confisqués avaient été adoptées en février 2000. La présente version à jour des lignes directrices prend note de l'évolution de la situation du trafic des espèces sauvages au cours des vingt dernières années. Tenant compte des dernières politiques et législations internationales, elle vise également à aider les gouvernements à prendre en charge le nombre toujours croissant d'animaux et de plantes vivants confisqués par les autorités et nécessitant leur intervention.

Bien que l'augmentation du nombre d'individus confisqués soit attribuable à des facteurs nombreux et complexes, elle est généralement liée à l'augmentation du trafic illicite des animaux et plantes vivants. De surcroît, les confiscations se multiplient également du fait que les autorités ont des connaissances plus pointues et comprennent mieux la situation. Dans certains cas, davantage de ressources sont désormais affectées aux autorités de confiscation et à leur agents. Les législations nationales et certains accords internationaux ont eux aussi fait l'objet de modifications, notamment la Convention sur le commerce international 
des espèces de faune et de flore sauvages menacées d'extinction (CITES). Un grand nombre de ces exigences légales sont devenues plus rigoureuses, soulignant toute l'importance de la gestion des confiscations d'espèces sauvages.

Sur le plan de la conservation de la biodiversité internationale, il est généralement nécessaire d'accorder la priorité à la prise en charge des plantes et des animaux appartenant à des espèces classées comme menacées d'extinction et dont la perte porterait préjudice aux individus en voie de disparition dans la nature. La faune et la flore étant soumises à une pression toujours croissante dans le monde entier, il est impératif de mettre en place, en matière de prise en charge des animaux et plantes confisqués, un processus de prise de décision au sein duquel la prévention

\section{de la disparition des espèces, le maintien des populations sau- vages et la conservation de la biodiversité passent par-dessus tout} autre critère et qui exige l'évaluation des risques courus, non seulement par les espèces confisquées, mais aussi par les espèces présentes dans les lieux envisagés pour la remise en liberté des individus captifs. Les lignes directrices de I'UICN ci-dessous ont été élaborées en pensant à ce besoin. Les espèces considérées comme menacées d'extinction sont celles classées vulnérables (VU), en danger (EN) ou en danger critique (CR) sur la liste rouge d'espèces menacées de l'UICN'M (http://www.iucnredlist.org/). De plus, il convient de définir les priorités aux fins de la prise en charge des individus confisqués appartenant à des espèces classées éteintes à l'état sauvage (EW), données insuffisantes (DD), quasi menacées (NT), non évaluées (NE) ou préoccupation mineure (LC) (des listes rouges nationales peuvent s'avérer utiles pour identifier les populations menacées d'espèces LC).

Les lignes directrices ci-dessous visent à aider les autorités gouvernementales à prendre des décisions en matière de prise en charge des animaux, plantes et champignons vivants confisqués en vertu des lois applicables à la faune et à la flore, quelle que soit leur valeur de conservation. Bien que les lignes directrices puissent également aider les autorités compétentes à prendre en charge les animaux et plantes appartenant à des espèces non menacées et ayant été confisqués, il convient de remarquer que l'un de leurs objectifs est le maintien de populations saines à l'état sauvage et que, de ce fait, les interventions visant uniquement à assurer le bien-être d'un individu particulier peuvent différer des actions préconisées. 


\subsection{Objectif des présentes lignes directrices}

Les présentes lignes directrices sont destinées aux instances gouvernementales et à leurs agents autorisés dont le mandat consiste à déterminer comment prendre en charge des organismes vivants confisqués dans l'immédiat (période initiale pouvant durer de quelques heures à quelques semaines) et à long terme (situation pouvant être qualifiée de «permanente»).

Aux fins du présent document, un spécimen est réputé confisqué dès qu'il est pris en charge par une instance compétente ou un agent responsable de décider de son sort à long terme. Ce terme ne s'applique donc PAS au moment de la capture, où d'autres protocoles de prise en charge sont susceptibles d'être en vigueur.

Remarque importante: si un organisme vivant inscrit aux annexes de la Convention CITES est confisqué et que le pays de confiscation est signataire de cette dernière, les protocoles y afférents en matière de prise en charge desdits spécimens priment sur les lignes directrices de l'UICN, notamment la résolution Conf. 17.8 sur l'utilisation des spécimens d'espèces inscrites aux annexes de la CITES commercialisés illégalement et confisqués.

\subsection{Principes de précaution}

L'un des plus grands principes des présentes lignes directrices est la prévention de toute perte de biodiversité à cause de remises en liberté inadéquates ou mal gérées. Parmi les principaux risques figurent la propagation de maladies dont sont atteints les animaux ou plantes relâchés, l'introduction d'animaux ou de plantes dans une zone dont ils ne sont pas originaires (avec le risque d'introduction d'espèces étrangères envahissantes ou d'introgression génétique, susceptibles d'entraîner une perte de biodiversité) et la concurrence anormale entre diverses espèces donnant lieu au déplacement d'espèces indigènes.

Les présentes lignes directrices se fondent sur le principe de précaution pour éviter que les risques évoqués ci-dessus et d'autres situations dangereuses ne se concrétisent. En particulier, l'autorité ayant confisqué l'organisme vivant doit s'abstenir de le relâcher dans la nature tant qu'elle ne dispose pas, pour l'animal ou la plante en question, d'un plan d'action convaincant qui contribue à la conservation de l'espèce ou ne présentant 
aucun risque significatif pour sa conservation dans son aire de répartition ou pour la conservation d'autres espèces dans le milieu de remise en liberté.

\subsection{Approche proactive}

Il peut être extrêmement difficile de prendre une décision propice à la conservation sans suffisamment d'informations, face à des arguments contradictoires ou devant des dilemmes d'éthique divergents soulevés par différents secteurs. De nombreux facteurs entrent souvent en jeu pour déterminer le mode de prise en charge d'un organisme vivant confisqué. Il peut donc être nécessaire de faire appel à des disciplines différentes pour obtenir les informations qui permettront d'aboutir à la décision idéale. De ce fait, les autorités chargées de la confiscation et leurs agents sont encouragés à établir des contacts aux niveaux local, national, régional et international, et à créer un réseau consultatif sur la confiscation (voir l'annexe 1) composé de spécialistes des domaines suivants:

- taxonomie, pour l'identification rapide et exacte des espèces/sous-espèces;

- médecine humaine et vétérinaire, pour la santé des êtres humains et des animaux, y compris pour la mise en quarantaine;

- sauvetage des animaux, élevage et éthologie;

- botanique;

- droit;

- logistique, pour orienter le stockage et le transport.

À cet égard, les autorités chargées de la confiscation sont encouragées à chercher à établir des points de contact avec:

- aux niveaux local, régional et international, les centres de sauvetage/ réadaptation des animaux, les zoos, les associations zoologiques et les sanctuaires pour animaux, qui sont susceptibles de fournir des conseils d'experts et, dans certains cas, d'héberger les organismes à court ou long terme;

- le bureau national de l'Organisation mondiale de la santé animale (OIE) et les départements de médecine vétérinaire des gouvernements/universités, qui sont susceptibles de fournir des conseils sur la santé et le bien-être des animaux;

- les jardins botaniques locaux, régionaux et internationaux; 
- l'organe de gestion et l'autorité scientifique de la CITES au niveau national, ainsi que le secrétariat CITES;

- les autorités policières et frontalières chargées de lutter contre les crimes sur la faune et la flore;

- d'autres instances et organismes consultatifs chargés de la santé de la faune et la flore et du bien-être des animaux, le cas échéant.

II est tout particulièrement important que les décisions prises par les autorités chargées de la confiscation et leurs agents soient en harmonie avec les décisions prises par les organes de gestion de la CITES. Toute possibilité de travail collaboratif entre ces deux instances (si elles sont séparées) doit être exploitée.

\subsection{Définitions}

Les définitions suivantes sont employées aux fins du présent document:

\section{Animaux:}

tout membre vivant de la faune reconnue, y compris les œufs.

\section{Organisme vivant confisqué:}

organisme vivant, dès sa prise en charge par une instance compétente ou un agent responsable de décider de son sort à long terme.

\section{Captivité/ex situ:}

situation dans laquelle des individus sont soumis à des contraintes spatiales ne correspondant pas à leur milieu naturel ou à celui de leurs progéniture, ou dans laquelle ils sont éloignés de leurs processus écologiques naturels et sont, dans une certaine mesure, pris en charge par des êtres humains. En bref, les individus sont conservés dans des conditions artificielles et soumis à des pressions de sélection différentes de celles existant dans un habitat naturel (lignes directrices de I'UICN sur la prise en charge ex situ pour la conservation des espèces).

\section{Pays d'origine du transit:}

pays depuis lequel un organisme a été transporté avant sa confiscation.

\section{Pays d'origine:}

pays dans lequel un spécimen/individu a été prélevé dans la nature, élevé en captivité ou reproduit artificiellement (glossaire terminologique de la CITES).

\section{Euthanasie:}

mise à mort d'un animal dans le but de mettre un terme à ses souffrances. 


\section{Mise à mort sans cruauté:}

la mise à mort sans douleur d'un animal dans un but autre que la cessation de ses souffrances [Remarque: les protocoles d'emploi de l'euthanasie et de la mise à mort sans cruauté, bien que ces deux méthodes soient utilisées pour des motifs très différents, sont présentés sous le terme " euthanasie » dans les lignes directrices de l'AMVA sur l'euthanasie].

\section{Aire de répartition indigène:}

distribution d'une espèce, connue ou déduite à partir d'informations (écrites ou verbales) historiques ou de preuves physiques d'occurrence de l'espèce (lignes directrices de l'UICN sur les réintroductions et les autres transferts aux fins de la sauvegarde).

\section{Plan unique:}

développement inclusif de stratégies de prise en charge et d'actions de conservation par les parties responsables pour toutes les populations d'une espèce, dans leur aire de répartition indigène ou en dehors.

\section{Plantes:}

tous les membres vivants de la flore reconnue, y compris les semences. Point de confiscation : point initial auquel une instance autorisée à pris en charge un organisme vivant.

\section{Renforcement:}

mouvement et remise en liberté intentionnels d'un organisme parmi une population existante dans le but de stimuler des populations d'espèces menacées dans la nature (lignes directrices de I'UICN sur les réintroductions et les autres transferts aux fins de la sauvegarde).

\section{Réintroduction:}

mouvement et remise en liberté intentionnels d'un organisme dans son aire de répartition indigène de laquelle il avait disparu (lignes directrices de l'UICN sur les réintroductions et les autres transferts aux fins de la sauvegarde).

\section{Rapatriement:}

retour d'un individu confisqué dans son pays d'origine.

\section{Transfert:}

mouvement d'organismes vivants facilité par l'être humain d'une zone vers une autre zone où ils sont remis en liberté, ce qui comprend les projets de réintroduction et de renforcement (lignes directrices de l'UICN sur les réintroductions et les autres transferts aux fins de la sauvegarde). 


\section{Critères de prise de décision}

Avant toute intervention, il est important de prendre en considération les trois critères prioritaires fixant les limites de la prise de décision en matière de prise en charge d'animaux et de plantes confisqués.

\subsection{L'échelle individuelle}

Le mode de prise en charge d'un organisme confisqué dépend en partie des attributs de l'individu en question, même s'il fait partie d'un grand nombre d'animaux ou de plantes confisqués. Une évaluation doit donc avoir lieu au niveau individuel. Sur le plan de la conservation, un facteur critique à analyser est l'espèce à laquelle appartient l'individu. Dans de nombreux cas, il ne sera pas possible de déterminer la population dont provient d'individu à l'état sauvage. Cependant, si cet élément est connu, les décisions doivent se fonder sur la population d'origine et non sur le pays d'origine. Une fois l'espèce identifiée, il est possible de dresser un plan d'action pour l'individu. Si l'espèce exacte n'est pas connue, les options de prise en charge en sont considérablement limitées. II peut s'avérer extrêmement difficile d'identifier un individu avec précision, notamment en présence d'un grand nombre d'animaux se ressemblant les uns aux autres. Pourtant, les décisions relatives au statut de conservation, à la santé et à l'origine, pour ne citer que celles-ci, nécessitent l'identification et le traitement de chaque organisme à titre individuel.

\section{Etude de casł marchés aux oiseaux indonésiens}

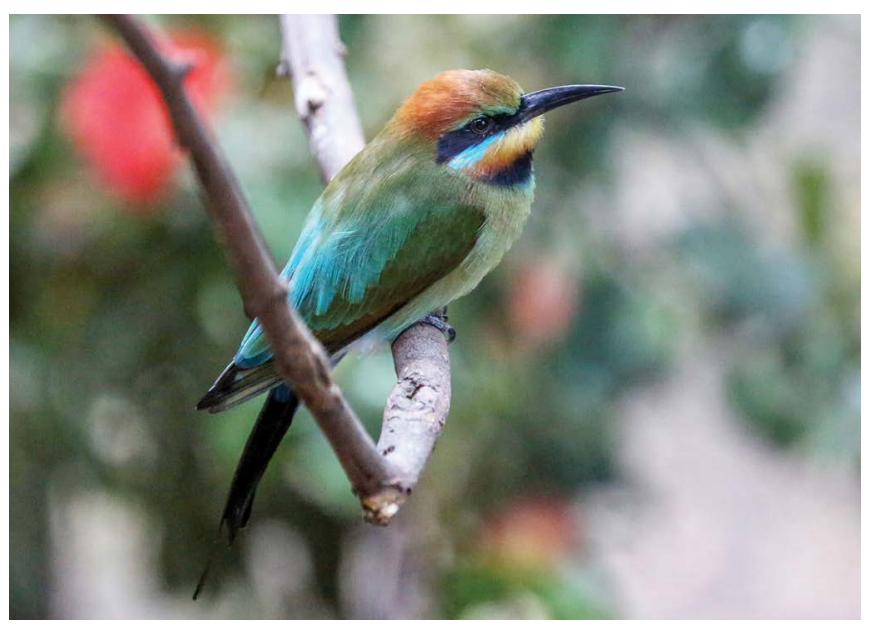

(c) Kira Mileham
Le marché aux oiseaux de Pramuka, à Djakarta, est l'un des plus grands d'Indonésie. De nombreux oiseaux ayant différents statuts de conservation sont capturés illégalement dans la nature et vendus sur le marché des oiseaux chanteurs. Faire la distinction entre les animaux vendus légalement et ceux qui ne le sont pas est une tâche colossale exigeant des compétences pointues et d'immenses ressources financières. À titre d'exemple, en 2016, suite à un sondage de trois jours, un rapport de TRAFFIC ${ }^{1}$ a fait état de près de 23000 oiseaux en vente sur cinq marchés à Surabaya, Yogyakarta et Malang, tout indiquant que la grande majorité d'entre eux avaient été prélevés illégalement dans la nature. De plus, un grand nombre des oiseaux vendus sur le marché aux oiseaux de Pramuka ne sont pas natifs, ont été importés illégalement et, s'ils ne sont pas pris en charge convenablement, pourraient constituer une grave menace à la vie des oiseaux indonésiens.

${ }^{1}$ Chng, S.C.L. et Eaton, J.A. (2016). In the Market for Extinction: Eastern and Central Java. TRAFFIC. Petaling Jaya, Selangor, Malaisie. 
Un organisme dont l'espèce n'est pas clairement connue (même si elle est soupçonnée) ne devrait JAMAIS être remis en liberté, car cela présenterait un risque inacceptable pour la biodiversité globale. Sauf circonstances atténuantes de poids (voir les lignes directrices de l'UICN sur les réintroductions et les autres transferts aux fins de la sauvegarde), en général, les individus doivent être relâchés SEULEMENT dans leur aire de répartition d'origine et, si elle est connue, parmi leur population d'origine. Ces restrictions sont nécessaires pour éviter l'introduction d'espèces envahissantes étrangères susceptibles de causer une pollution génétique et d'altérer la structure génétique des espèces présentes.

\subsection{Ressources disponibles}

L'un des facteurs les plus importants influençant la prise de décisions est le niveau de ressources dont dispose l'autorité chargée de la confiscation. Naturellement, le niveau de ressources peut varier en fonction des circonstances et des priorités de la conservation. Cependant, il existera dans chaque cas une quantité déterminée de ressources et les autorités seront amenées à évaluer les actions souhaitables, adéquates et possibles au regard des contraintes budgétaires. Les autorités chargées de la confiscation peuvent confier la prise en charge des individus confisqués à une organisation tierce responsable, et le niveau de ressources dont cette dernière dispose affectera inévitablement le mode de prise en charge de ces individus à court et à long terme. Avant de choisir une option de prise en charge particulière, il convient de soulever les questions suivantes sur la disponibilité des ressources:

i. Existe-t-il suffisamment de ressources disponibles (fonds, personnel, capacité de mise en quarantaine, savoir-faire) pour le rapatriement de l'individu vers le pays/la région d'origine et pour sa prise en charge dans le respect des normes établies dans les présentes lignes directrices?

ii. Existe-t-il suffisamment de ressources disponibles pour la mise en œuvre d'un programme de réintroduction/transfert/renforcement (fonds, savoir-faire, suivi), surtout pour les espèces classées menacées?

iii. Existe-t-il suffisamment de ressources disponibles pour assurer la prise en charge à long terme ex situ (c'est-à-dire en captivité) de l'individu tout en assurant son bien-être de manière adéquate (fonds, infrastructures, 
personnel, savoir-faire)?

iv. Existe-t-il une organisation ou un groupe d'organisations compétent susceptible de fournir les ressources nécessaires aux actions ci-dessus, et donc de servir de mandataire (de l'autorité ayant confisqué l'individu et du pays d'origine) chargé de la prise en charge de l'individu?

v. Le niveau de ressources affecté correspond-il à la valeur de l'individu? L'UICN est conscient que la disponibilité des ressources est parfois le plus important facteur de prise de décision. Cependant, lorsque l'individu pris en charge appartient à une espèce classée menacée, il faudra parfois déployer des efforts considérables pour obtenir les ressources requises.

\subsection{Facteurs juridiques et conventions internationales}

Les décisions relatives au mode de prise en charge d'un individu dépendent, non seulement des propriétés de ce dernier et des ressources disponibles, mais aussi des conditions locales, et notamment des lois en vigueur dans le pays où il a été confisqué et des conventions, réglementations ou accords internationaux. Dans le contexte des confiscations, si l'autorité se trouve dans un pays tenu aux réglementations de la CITES, le protocole de prise en charge des espèces inscrites aux annexes de la CITES (résolution Conf. 17.8 de la CITES sur l'utilisation des spécimens d'espèces inscrites aux annexes de la CITES commercialisés illégalement et confisqués) doit être respecté et appliqué par l'organe de gestion. 


\subsection{Soins immédiats à court terme}

Dans le cadre de la prise en charge d'un organisme confisqué, la première phase de l'intervention consiste à veiller à la sécurité de l'individu et, dans la mesure du possible, au soulagement de ses souffrances. De nombreux animaux et plantes confisqués par les autorités souffrent de différentes façons : détresse, manque de nourriture, d'eau ou de lumière, piètres conditions de transport, maladie et mauvaises pratiques d'élevage. La plus grande priorité devrait être d'apporter aux animaux et aux plantes des soins adéquats en urgence pour répondre à leurs besoins les plus élémentaires: eau, nourriture, espace, lumière et abri. Différentes espèces ont différents besoins en matière d'élevage et de bien-être. Cela est le cas tant pour les animaux que pour les plantes. Toute négligence à cet égard (par exemple si une plante succulente est arrosée excessivement) risque d'intensifier leurs souffrances et d'augmenter le taux de mortalité. Par conséquent, comme indiqué ci-dessus, il est vivement recommandé aux autorités de consulter, voire de créer elles-mêmes, un réseau consultatif sur la confiscation pour recevoir des conseils sur les soins à apporter dans l'immédiat, à court terme. L'annexe 1 définit le mandat qui pourrait être confié à un tel réseau consultatif sur la confiscation. Des renseignements supplémentaires sont disponibles dans le document World Zoo and Aquarium Animal Welfare Strategy.

En raison du risque de contagion à d'autres organismes (y compris à des êtres humains), il est impératif de mettre immédiatement les organismes confisqués en quarantaine. Les éléments nécessaires à la mise en quarantaine peuvent grandement varier selon les espèces et les circonstances. Une orientation sur les procédures à suivre et les infrastructures à utiliser est fournie dans le Manuel de procédures pour l'analyse du risque de maladies chez les animaux sauvages de l'OIE-UICN. Le Code sanitaire pour les animaux terrestres de l'OIE, chapitre 5.6, et les Protocoles de mise en quarantaine et de contrôle de santé préliminaire au transfert et à la remise en liberté de l'OIE-UICN-EAZWV fournissent eux aussi des informations complémentaires utiles. Les liens vers ces documents et vers d'autres documents utiles figurent dans l'annexe 2.

Une fois les premiers besoins satisfaits, il est nécessaire de recueillir des informations pour orienter le processus de prise de décision et de prise en charge à long terme. 
Les sections suivantes doivent être utilisées avec les diagrammes suivants: collecte d'informations et évaluation initiale (annexe 3) et arbre décisionnel - espèces confisquées (annexe 4).

\subsection{Processus de collecte d'informations et d'évaluation initiale}

\subsubsection{Identification des espèces}

Une fois que l'organisme se trouve en sécurité, il est impératif que l'autorité détermine l'espèce précise de chaque individu. Cette tâche peut être ardue, surtout si un grand nombre d'individus relevant de taxons aux apparences similaires ont été confisqués, D'où l'importance de nouer proactivement des liens avec des experts de la taxonomie qui pourront apporter leur concours dans le cadre d'un réseau consultatif sur la confiscation (voir l'annexe 1).

\section{Étude de cas: identification d'espèce}

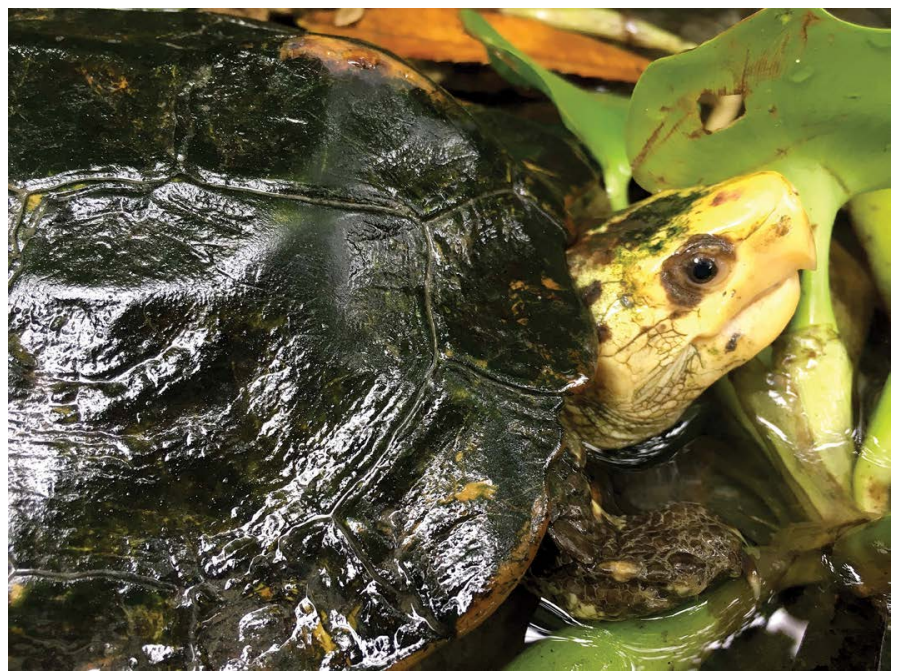

La tortue boîte d'Indochine (Cuora galbinifrons) est originaire du Nord du Vietnam, du Nord du Laos et du Sud de la Chine. La tortue boîte de Bouret (Cuora bouretti) occupe l'écosystème équivalent dans les régions centrales du Vietnam et du Laos. En 2004, des analyses ADN ont abouti au reclassement de la tortue boîte de Bouret, qui était auparavant une sous-espèce de la $C$. galbinifrons, en une espèce à part entière. De l'extérieur, ces deux espèces sont pratiquement impossibles à distinguer. Elles figurent toutes les deux dans les listes d'espèces menacées (CR), sont très prisées dans le commerce illégal et sont traquées par les braconniers. Après avoir été capturées par des braconniers et transportées, ces espèces peuvent être mélangées dans la même caisse et devenir très difficile à différencier, à moins qu'un expert de l'herpétologie ne soit présent.

Si ce travail n'est pas réalisé avec le plus grand soin, compte tenu de la ressemblance visuelle existant entre de nombreuses espèces d'animaux et de plantes, le risque de méprise est élevé et peut faire dérailler toutes les décisions ultérieures. L'identification peut s'avérer encore plus compliquée si l'on tient compte de la variété des sous-espèces et des changements taxonomiques.

C'est souvent aux autorités ayant confisqué l'individu qu'il appartient de l'identifier. II est vivement recommandé de consulter les guides, manuels et légendes taxonomiques disponibles dans le cadre du travail d'identification élémentaire. Toute erreur d'identification peut être lourde de conséquences 
et, de ce fait, il est recommandé que, en cas de doute ou de confusion, les autorités fassent appel à des spécialistes de la taxonomie. Ces derniers peuvent travailler dans des musées d'histoire naturelle, des universités, des herbiers, des jardins botaniques, des zoos ou des aquariums. Certains savants peuvent également être réputés pour leur érudition en rapport avec un taxon particulier. Le groupe de spécialistes de la Commission de sauvegarde des espèces de l'UICN (CSE) peut parfois apporter l'assistance requise ou mettre ceux qui le sollicitent en rapport avec les experts demandés. Les experts en taxonomie peuvent souvent apporter leur aide à ce processus d'identification grâce aux images numériques, bien que, dans certains cas, il soit nécessaire de réaliser un profilage ADN pour établir l'identité de l'animal de façon incontestable,

3.2.2. Statut de conservation et priorités de I'UICN

La liste rouge des espèces menacées de I'UICN'M a grandement contribué à l'identification du statut de conservation des espèces connues et, bien qu'elle ne soit pas complète, elle constitue un excellent point de départ pour les autorités chargées de la confiscation.

Dans l'intérêt de la conservation de la biodiversité mondiale, dans le cadre de la prise en charge des organismes confisqués, nous estimons que les ressources disponibles devraient être affectées en priorité aux individus appartenant à des espèces menacées d'extinction ou dont la valeur de conservation est inconnue. II s'agit notamment des espèces classées en danger critique, en danger, vulnérables, insuffisance de données, non évaluées ou éteintes à l'état sauvage.

Au-delà de la liste rouge internationale de l'UICN, il convient de consulter les listes rouges nationales et régionales (lorsqu'elles existent), les plans d'action nationaux pour la biodiversité et les classements d'espèces menacées des autorités nationales pour déterminer la valeur de conservation régionale d'un individu confisqué. L'identification d'individus appartenant à des espèces nationalement importantes peut également nécessiter des connaissances et ressources spécialisées.

\subsubsection{Santé}

Qu'ils soient en transit, en stockage ou en cours de transfert, les animaux et les plantes déplacés en dehors de leur aire de répartition indigène peuvent présenter un risque considérable de transmission de maladies pour 
les êtres humains et leurs moyens de subsistance, ainsi que pour d'autres individus de la même ou d'autres espèces. L'atténuation de ces risques fait l'objet de l'analyse du risque de maladies chez les animaux sauvages de I'UICN et des lignes directrices de l'UICN sur les réintroductions et les autres transferts aux fins de la sauvegarde.

Il est crucial de réaliser une évaluation des possibilités de survie de l'organisme individuel, à l'état sauvage ou en captivité (à court et long terme), et notamment de déterminer s'il ne souffre d'aucune maladie susceptible d'infecter des populations sauvages ou captives de la même ou d'autres espèces. Par ailleurs, l'appréciation des possibilités de survie va souvent bien au-delà d'un simple bilan de santé immédiate. II n'est pas rare que des individus confisqués soient physiquement ou (pour les animaux) mentalement incapables de vivre sans les soins qui leur ont été apportés en captivité et, dans certains cas plus extrêmes, l'euthanasie de l'animal ou la destruction de la plante peuvent être les seules options. II est parfois tout aussi impossible de remettre en liberté des phénotypes hybrides ou inhabituels.

Dans les présentes lignes directrices, le terme «euthanasie» désigne le fait de mettre un terme à la vie d'un animal blessé ou malade, sans cruauté, dans l'intention d'abréger ses souffrances. Les protocoles d'exécution de l'euthanasie d'un animal blessé ou malade sont définis dans les lignes directrices de l'AMVA sur l'euthanasie.

II n'est pas aisé de déterminer les possibilités de survie et, pour tout individu confisqué, les autorités devront probablement faire appel à des experts dans le cadre d'un réseau consultatif sur la confiscation (voir l'annexe 1).

3.2.4. Possibilités de survie Les possibilités de survie de l'individu dans la nature doivent être analysées et, pour ce faire, il convient de se demander s'il a déjà existé à l'état sauvage, sans aucune assistance, ou s'il serait en mesure de le faire. Certains individus nés ou ayant vécu une grande partie de leur vie en captivité, ou ayant été reproduits artificiellement, ont peut-être sans assistance et de contribuer à la conservation de la biodiversité globale. Cependant, ce n'est pas toujours le cas et un spécialiste de l'éthologie, de l'élevage ou de la botanique devra se pencher sur cette question dans le cadre d'un réseau consultatif sur la confiscation (voir l'annexe 1) avant qu'une décision ne soit prise. Si l'organisme est né en captivité ou a été artificiellement repro- 
duit, mais est jugé apte à être remis en liberté, les lignes directrices de I'UICN sur les réintroductions et les autres transferts aux fins de la sauvegarde et les lignes directrices de l'UICN sur la prise en charge ex situ pour la conservation des espèces doivent être utilisées pour déterminer si la remise en liberté de l'individu favorisera la conservation, avec un niveau de risque minimal et acceptable pour les populations sauvages.

\subsubsection{Motif de confiscation}

Dans certains cas, la confiscation peut être temporaire, peut-être parce que les documents requis n'ont pas été remplis correctement pour le transfert de l'individu. Une confiscation peut donc avoir lieu sur le court terme (protocole de prise en charge jusqu'à ce que l'individu puisse être déplacé légalement) ou sur le long terme (protocole de prise en charge pour un individu confisqué en raison d'obstacles juridiques insurmontables). En rapport avec le motif de confiscation, deux questions doivent être soulevées:

\section{i. Pourquoi l'individu a-t-il été confisqué?}

S'agit-il d'une formalité non remplie pour une espèce pouvant être légalement commercialisée, ou est-on en présence d'une espèce dont le commerce est interdit en vertu de conventions internationales et de lois nationales?

Si l'individu appartient à une espèce pouvant être légalement commercialisée, le «seul» problème concerne la légalité de l'acquisition, de l'opération commerciale ou de la détention. En fonction de la situation et de la région,

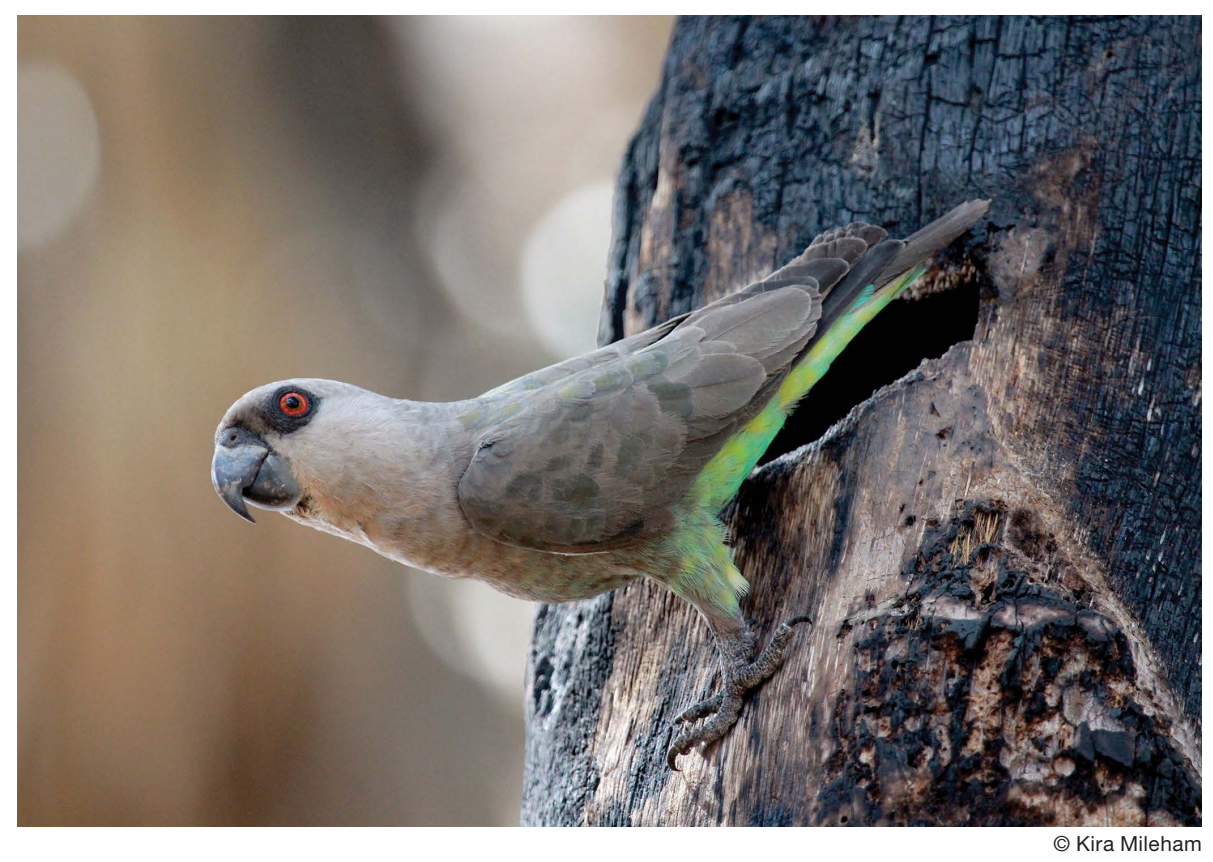


plusieurs possibilités s'offrent aux autorités, par exemple garder l'organisme individuel jusqu'à ce que toutes les formalités aient été remplies, ou le prendre en charge de façon continue.

\section{ii. Qui revendique l'individu?}

Qui prétend être le propriétaire de l'individu? Si cette personne est connue, la question du motif de la confiscation se pose de nouveau. En fonction de la situation, plusieurs possibilités s'offrent aux autorités, par exemple garder l'organisme individuel jusqu'à ce que toutes les formalités aient été remplies, ou le prendre en charge de façon continue. Si personne ne revendique l'individu, l'arbre décisionnel permettra de déterminer le processus de prise en charge à suivre (voir l'annexe 4).

3.2.6. Pays d'origine et d'arrivée

II peut être extrêmement difficile de déterminer le pays d'origine d'un individu, surtout si celui-ci a traversé plusieurs régions ou pays avant sa confiscation. De plus, certaines espèces et sous-espèces sont présentes à l'état sauvage dans de nombreux pays. Par exemple, l'identification du pays d'origine d'un perroquet jaco et d'un perroquet timneh (Psittacus erithacus et Psittacus timneh) confisqués peut s'avérer problématique, car cette espèce commercialisée est native dans treize pays et passe souvent les frontières clandestinement.

Sur le plan de la prise en charge, l'idéal est de confisquer les individus dans leur pays d'origine. Le processus de prise en charge s'en voit grandement simplifié, car chaque pays est responsable de gérer ses propres ressources en matière de biodiversité et l'État propriétaire de l'individu est identifié sans problème. Le processus de prise en charge et les options de prise en charge se compliquent lorsque l'individu est confisqué ailleurs que dans le pays d'origine. Quoi qu'il en soit, il est important de déterminer si le pays d'origine se trouve ou non dans l'aire de répartition indigène de l'espèce, si l'individu appartient à une espèce étrangère envahissante dans son pays d'origine, ou s'il n'est ordinairement pas présent (naturellement ou comme espèce étrangère envahissante) dans le pays d'origine. Ces différents scénarios ont des impacts différents sur les décisions de prise en charge future de l'individu en question.

3.2.7. Espèces envahissantes reconnues

Certaines espèces sont reconnues comme présentant un sérieux potentiel envahissant et donc une menace importante à la biodiversité globale. Elles sont inscrites dans la base de données mondiale sur les espèces 
envahissantes de I'UICN. Les individus appartenant à ces espèces ne devraient jamais être relâchés dans la nature en dehors de leur aire de répartition indigène.

\subsection{Détention provisoire}

Une fois que suffisamment d'informations ont été recueillies pour prendre une décision éclairée, il peut s'avérer nécessaire de garder pendant un certain temps un organisme vivant, dans des conditions adéquates assurant son bien-être, avant qu'il n'atteigne sa destination finale. Dans un tel cas, il faut trouver un lieu de détention provisoire avec l'aide d'un réseau consultatif pour la confiscation (voir l'annexe 1). Les soins prodigués doivent correspondre à la norme définie dans le document Caring for Wildlife: The World Zoo and Aquarium Animal Welfare Strategy.

Toutes les informations ci-dessus ayant été recueillies, il est possible de prendre une décision de prise en charge à long terme à l'aide de l'arbre décisionnel (voir le diagramme dans l'annexe 4).

\subsection{Arbre décisionnel pour la prise en charge à long terme}

Trois possibilités s'offrent aux autorités chargées de la confiscation, avec une quatrième option moins désirable si la loi le permet, dans les cas où les trois premières possibilités ne seraient pas faisables. Toutes les décisions doivent être transparentes et fondées sur des éléments probants clairs. Ces possibilités sont les suivantes:

1. Rapatriement;

2. Transfert de conservation (y compris réintroduction);

3. Prise en charge ex situ à long terme; et

4. Mise à mort sans cruauté/destruction (si les conditions locales le permettent) des individus aptes (animaux ou plantes) ou euthanasie des animaux inaptes

Remarque importante : Veuillez consulter les diagrammes (annexes 3 et 4) pour déterminer la possibilité la mieux adaptée à un individu particulier.

\subsubsection{Rapatriement}

Si la confiscation n'a pas eu lieu dans le pays d'origine, les autorités ayant confisqué l'individu peuvent envisager de le renvoyer vers son pays d'origine pour que les autorités de ce pays décident de son sort. Certains 
facteurs importants doivent toutefois être examinés avant de prendre une décision de rapatriement. Les questions suivantes doivent être soulevées:

- Le pays d'origine se trouve-t-il dans l'aire de répartition indigène de l'espèce?

- Sait-on si le pays d'origine du transit est le pays d'origine de l'individu? ॥ arrive parfois que, avant d'être confisqué, un individu ait traversé plusieurs pays et que le pays d'origine ne soit pas connu.

- Le pays d'origine est-il disposé à et capable de prendre en charge l'individu confisqué?

- Le rapatriement pose-t-il certains problèmes quant au bien-être de l'individu ? Si tel est le cas, il vaudrait mieux envisager une autre option.

- Les parties responsables de supporter les frais du rapatriement ont-elles été mutuellement convenues?

- Le rapatriement vers le pays d'origine est-il dans l'intérêt de l'individu (notamment en cas de guerre civile, de contraintes économiques, etc.)?

- Le pays compte-t-il des experts/des ONG reconnus capables d'aider les autorités à prendre les individus rapatriés, au besoin?

\section{Étude de casł remise en liberté inadéquate aboutissant à l'expansion d'une espèce étrangère envahissante}

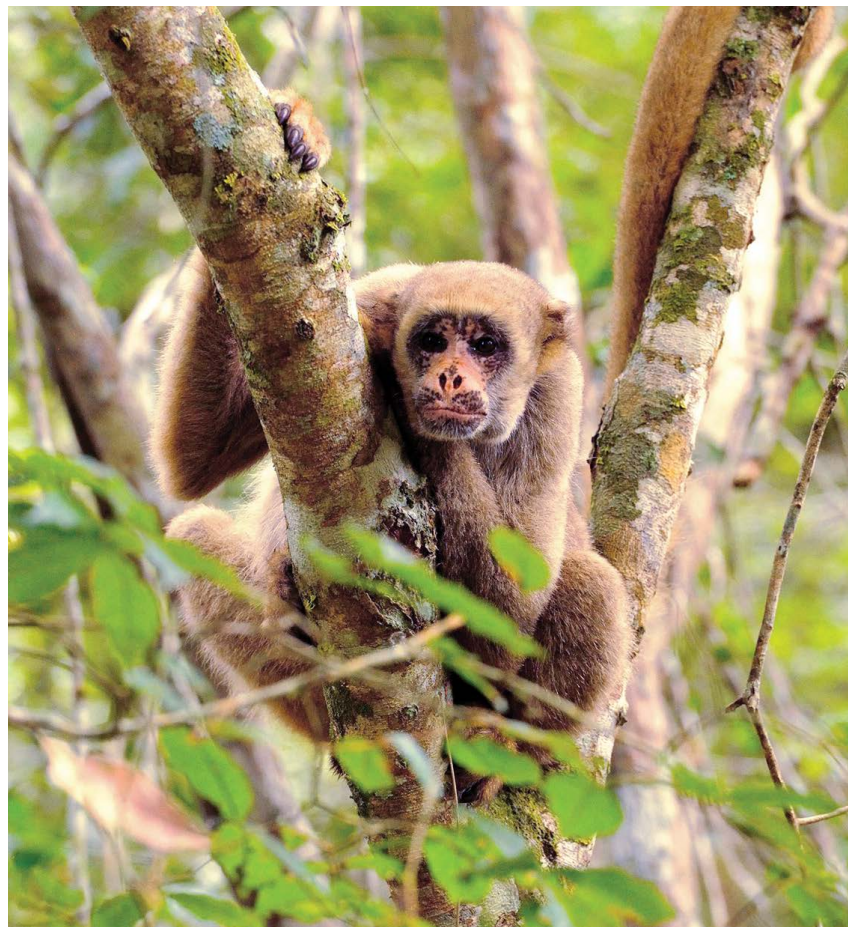

(C) Russell A. Mittermeier
Informations tirées de «Of Least Concern? Range extension by Rhesus Macaques (Macaca mulatta) threatens long-term survival of Bonnet Macaques (M. radiata) in peninsular India». Par Kumar, R., Radhakrishna, S. et Sinha, A. (2011) International Journal Primatology 32: 945-959. doi:10.1007/s10764-011-9514-y

«Les macaques à bonnet ont été déplacés de nombreuses zones de leur ancienne aire de répartition. Les limites distributionnelles du sud et du nord pour les macaques rhésus et les macaques à bonnet, respectivement, sont parallèles dans la partie occidentale du pays, étant séparées par un vaste espace au centre de I'Inde, puis convergent sur la côte orientale pour former une zone de chevauchement de distribution. Cette région de chevauchement se caractérise par la présence de troupes mixtes, certaines troupes ne comportant qu'une seule espèce se trouvant parfois même à proximité les unes des autres. La vaste aire de répartition des macaques rhésus, issue des processus naturels à certains endroits mais, dans d'autres régions, résultant directement de l'introduction de cette espèce, a des conséquences graves pour la populations endémiques des macaques à bonnet, qui sont en déclin en Inde méridionale.» 
- Dispose-t-on de garanties suffisantes indiquant que le pays d'origine dispose d'un système efficace pour éviter que l'individu confisqué ne retombe dans le commerce illégal?

3.4.2 Transfert (ou restitution de l'individu confisqué dans la nature)

Cette possibilité peut être la plus attrayante pour les autorités, car non seulement elle semble favoriser le bien-être de l'individu (de l'animal), mais elle est aussi généralement bien perçue par le grand public et est souvent considérée, sur le plan moral, comme «la chose juste». II existe néanmoins plusieurs facteurs très importants à prendre en considération avant de choisir une telle option, même avec les meilleures intentions.

En effet, la restitution d'un individu dans la nature de façon responsable peut être extrêmement difficile, nécessite souvent un engagement durable et exige des ressources considérables (financières et autres). L'autorité ayant confisqué l'individu doit donc être certaine de son engagement et de la disponibilité des ressources nécessaires à la réussite d'une telle action. Ceci dit, sur le plan de la conservation, le transfert d'une espèce peut être un outil très précieux. II convient de remarquer que, compte tenu de l'importance de la conservation de la biodiversité et des ressources limitées (financières et autres) devant être réparties entre divers projets, le transfert d'individus confisqués d'une espèce non menacée ne doit pas primer sur celui d'espèces menacées. Dans la pratique, en raison des contraintes existant dans de nombreux pays dans le domaine de la confiscation, cela signifie que les ressources doivent d'abord être affectées aux transferts visant une finalité de conservation et non les transferts dont le motif est lié au bien-être, à des sensibilités religieuses ou esthétiques, à des facteurs budgétaires ou au côté pratique.

Par ailleurs, le transfert d'espèces non natives est incompatible avec les principes de conservation de la biodiversité et, de ce fait, une espèce dont l'origine est inconnue, qui n'est pas indigène ou dont le risque de maladie n’a pas été déterminé ne doit en aucun cas être remise en liberté.

Les lignes directrices de l'UICN sur les réintroductions et les autres transferts aux fins de la sauvegarde fournissent une orientation approfondie permettant d'évaluer le bien-fondé des transferts à des fins de sauvegarde en tant qu'outil de prise en charge des individus confisqués. 


\subsubsection{Prise en charge ex situ à long terme}

Un certain nombre de raisons peuvent justifier qu'un individu ne puisse

pas être relâché dans la nature, que ce soit immédiatement ou à long terme, et qu'il soit donc nécessaire de le garder en captivité de façon durable. Ces raisons sont notamment, sans toutefois s'y limiter, les suivantes:

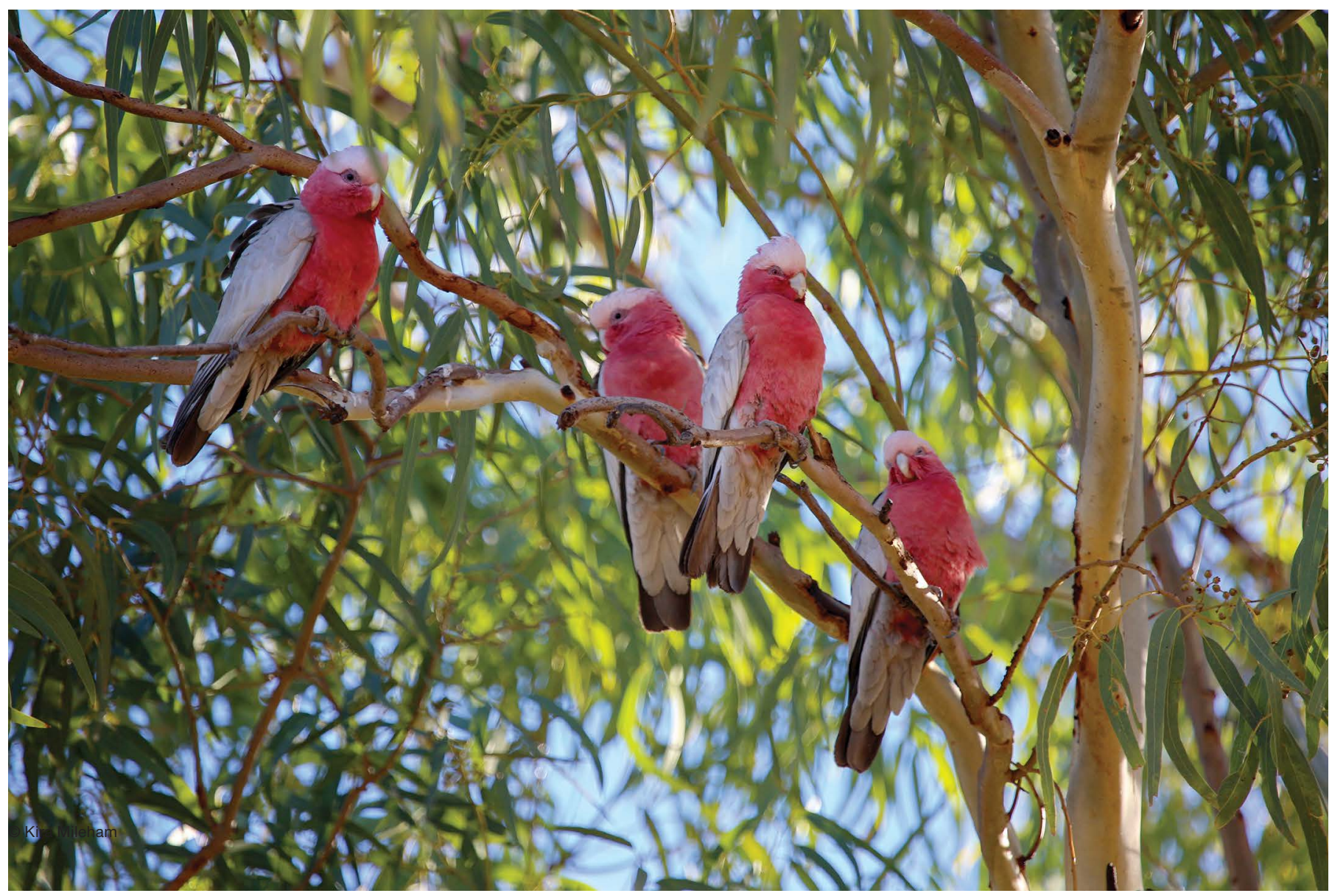

- Il n'y a pas assez d'informations disponibles, par exemple sur l'identification de l'espèce ou le risque de maladie, pour permettre le transfert.

- L'âge, la phase du cycle de vie de l'individu ou d'autres raisons indiquent qu'il ne dispose pas des aptitudes nécessaires à sa survie dans la nature.

- La santé physique ou psychologique de l'individu n'est pas optimale.

- Les besoins écologiques de l'espèce à laquelle appartient l'individu (p.

ex. conditions requises pour son habitat, limites de capacité de transport, etc.) rendent un transfert difficile.

- Les besoins sociaux/comportementaux de l'espèce à laquelle appartient l'individu (p. ex. pyramide des âges, rapport des sexes, structure sociale, etc.) rendent un transfert difficile.

- II n'existe pas assez de ressources disponibles pour mettre en œuvre un 
programme de remise en liberté.

- Aucun habitat adéquat n'est disponible.

- L'individu n'est pas représentatif de sa forme à l'état sauvage (p. ex. hybride, couleur non présente dans les morphologies à l'état sauvage, etc.).

- Les exigences requises pour l'obtention du permis ou le retard de délivrance des autorisations gouvernementales rendent le transfert difficile.

- La survie de l'espèce fait face à des menaces immédiates (telles que la chasse, les conflits humains/animaux en raison desquels une espèce est considérée comme «nuisible»).

- Il existe un risque pour d'autres espèces, voire pour la santé publique.

Si la détention en captivité à long terme est l'option privilégiée, les lignes directrices de I'UICN pour la prise en charge ex situ pour la conservation des espèces devraient être étudiées avec attention pour comprendre l'impact d'une telle décision sur le plan de la conservation. La World Zoo and Aquarium Animal Welfare Strategy doit elle aussi être consultée pour comprendre les approches et les soins à mettre en œuvre pour que cette décision soit viable sur le long terme. Si les conditions requises pour la détention à long terme de l'individu sont remplies, les présentes lignes directrices doivent servir d'outil de prise en charge continue.

Dans certains pays, la loi exige que les individus confisqués appartenant à des espèces non aptes au transfert soient placés dans des établissements de détention (tels que des centres d'accueil, des zoos ou des jardins botaniques). Cela risque d'imposer une charge à ces centres et conduire à des difficultés en matière de ressources ou de bien-être. Dans certains cas, le retour d'individus appartenant à des espèces non menacées dans le commerce légal peut être une solution de prise en charge envisageable, sous réserve que cela n'ait PAS un effet indésirable sur les populations sauvages d'espèces menacées (par exemple en stimulant un commerce illégal ou non durable de cette espèce ou d'espèces semblables, au niveau national ou international, en propageant des maladies, ou encore ou introduisant l'espèce en dehors de son aire de répartition indigène). Un retour dans le commerce légal peut uniquement être permis si les autorités de contrôle sont fortes et efficaces, de façon à ne pas encourager la corruption et le commerce illégal, et à ne pas aider des personnes physiques ou morales à profiter du commerce illégal.

II convient de remarquer que la détention d'individus à long terme présente des risques comme des avantages. Parmi les risques pour les animaux, les plantes et les êtres humains figurent le danger de maladie, les évasions et 
les frais liés aux soins en captivité. Pour ce qui est des avantages, ces individus peuvent être utilisés pour l'élevage contrôlé, pour les programmes éducatifs et pour la recherche.

Si la captivité à long terme est choisie, il convient de privilégier le placement des individus confisqués dans des zoos et des jardins botaniques, qui disposent de l'espace et du savoir-faire nécessaires, et qui participent à des programmes d'élevage nationaux ou internationaux ex situ dans le cadre d'une approche de plan unique.

3.4.4 Mise à mort sans cruauté/destruction

La mise à mort sans cruauté est, en tant qu'outil de prise en charge consistant à tuer l'animal pour des raisons autres que le soulagement de la souffrance (euthanasie), interdite dans de nombreux pays et considérée comme inacceptable pour des motifs religieux ou éthiques dans d'autres pays. Les lois et la culture locales doivent être respectées pour déterminer s'il s'agit d'une option disponible.

Bien que la mise à mort sans cruauté puisse être perçue comme un échec de toutes les autres possibilités de prise en charge, il est parfois la seule option adéquate permettant d'éviter certains problèmes par la suite, par exemple lorsque l'individu risque d'être maltraité ou relâché de façon irresponsable.

La manière dont un pays prend en charge ses animaux peut avoir un impact significatif sur la façon dont la faune et la flore y sont perçues. D'importants facteurs juridiques, éthiques et moraux doivent être pris en considération par les autorités chargées de la confisqué (ou par leurs mandataires) avant d'avoir recours à la mise à mort sans cruauté comme outil de prise en charge approprié pour les animaux. II ne faut pas l'utiliser à la légère. Si toutes les autres possibilités ont été examinées en détail et que la mise à mort sans cruauté est justifiable, la procédure doit être dénuée de toute cruauté. Les lignes directrices de l'AMVA sur l'euthanasie fournissent une orientation en la matière.

La destruction de plantes vivantes confisquées appartenant à des espèces non menacées est recommandée aux autorités comme outil approprié si les autres possibilités ont été écartées comme inadéquates. En raison du risque présenté par les espèces envahissantes de plantes vivantes confisquées (y compris les semences), l'incinération de toutes les parties de la plante confisquée est la méthode de destruction recommandée. 


\section{Conclusion}

Comme indiqué précédemment, aucune de ces options ne saurait être considérée comme parfaite à tous égards. En réalité, l'idéal serait d'empêcher le commerce illégal des animaux et plantes vivants à la source. Cependant, nous vivons dans un monde imparfait et les autorités chargées de la confiscation sont donc amenées à prendre des décisions difficiles. Les présentes lignes directrices sont fournies pour aider ces autorités à soupeser les facteurs liés à la conservation dans le cadre de leurs décisions.

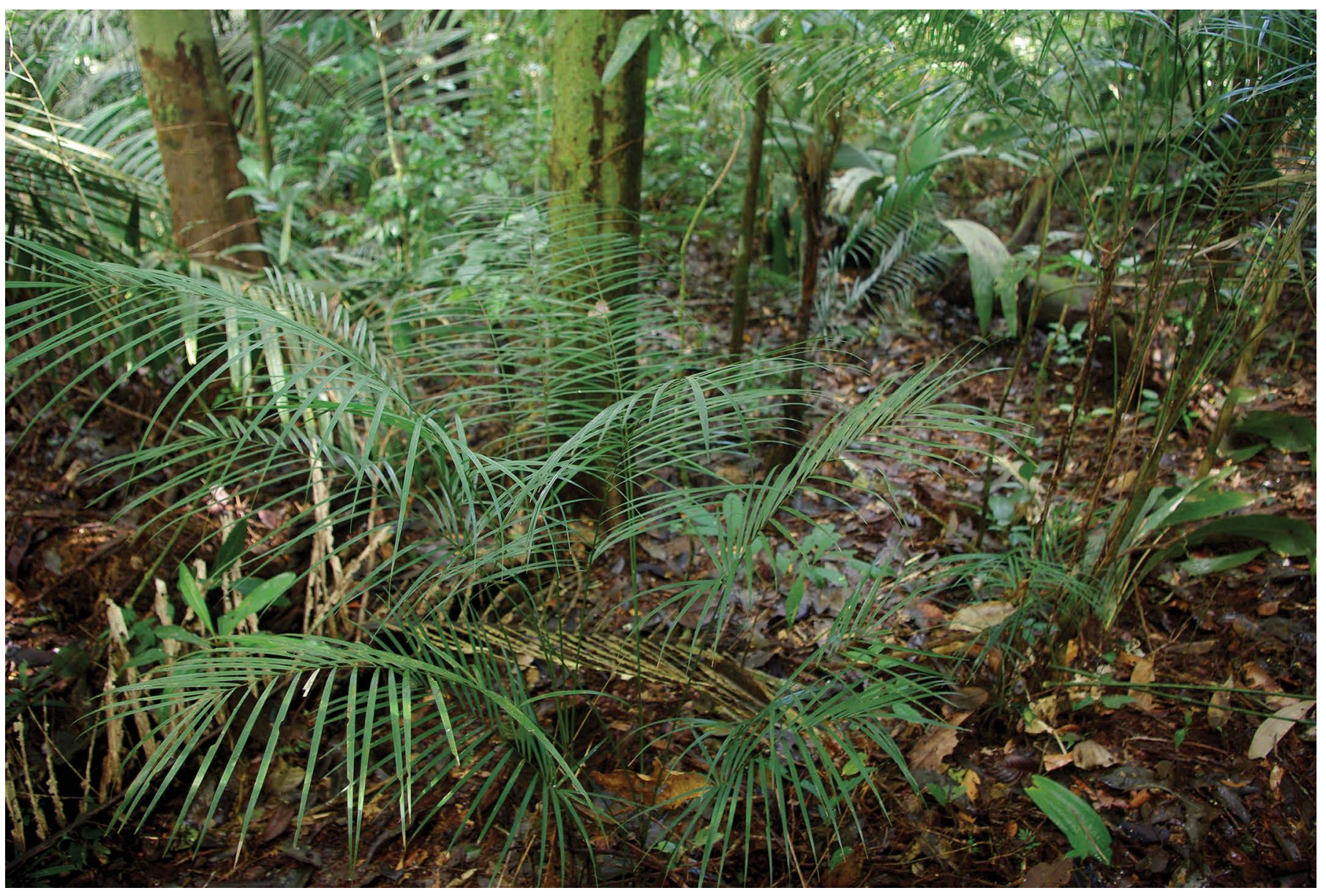

() Thomas Couvreur 



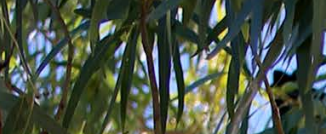

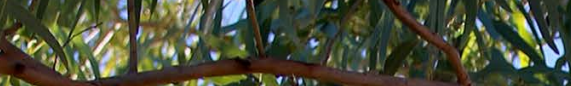

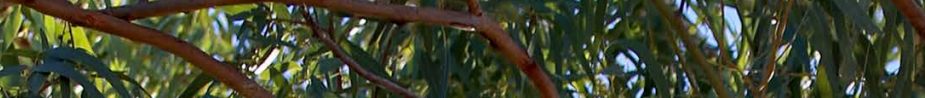
(ip.

if:

4

(i)

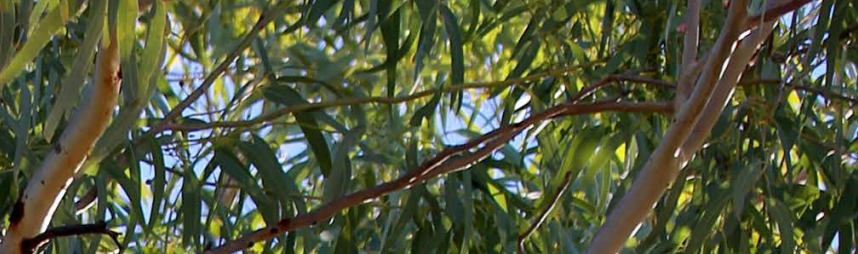

tor

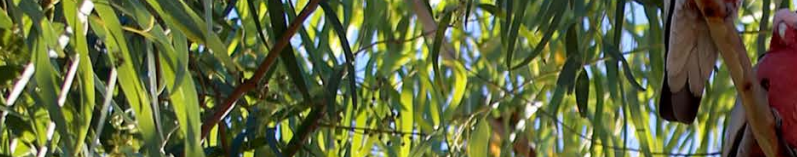

10) 2 is

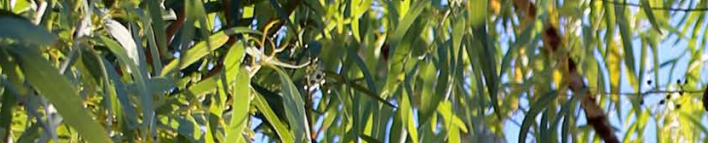

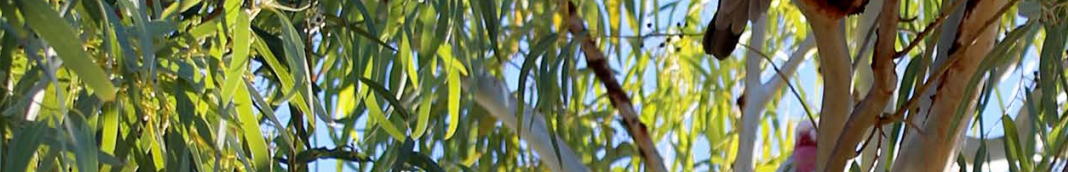

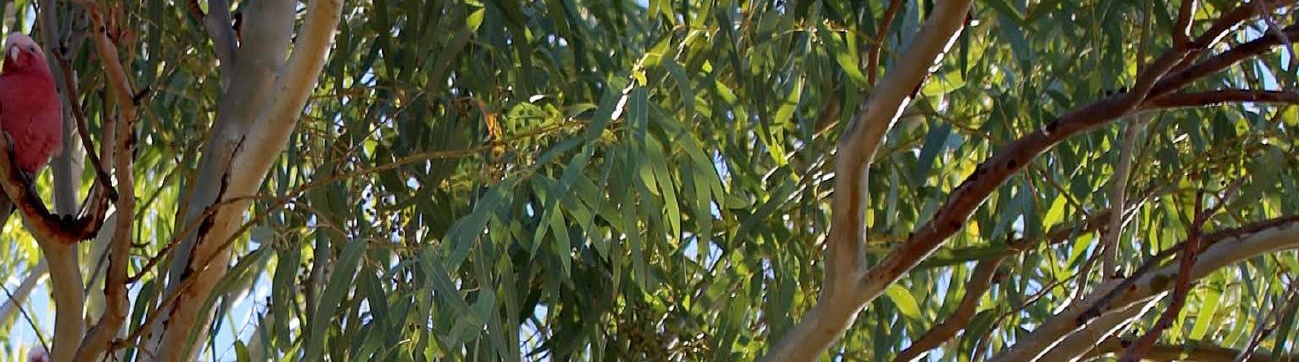

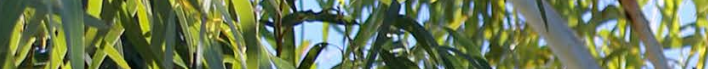

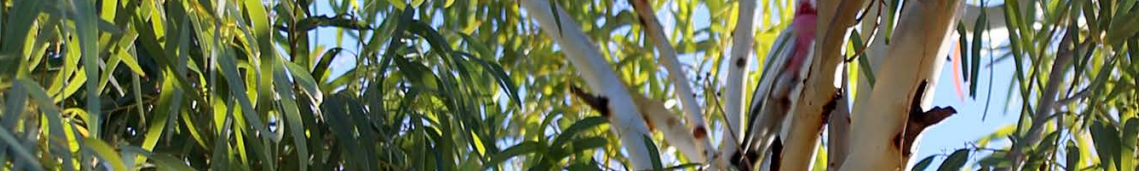

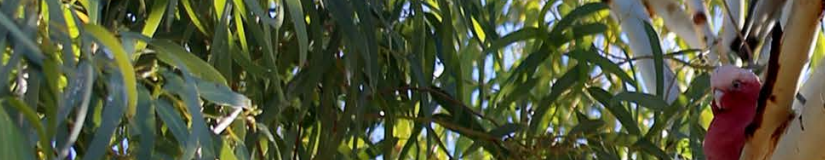

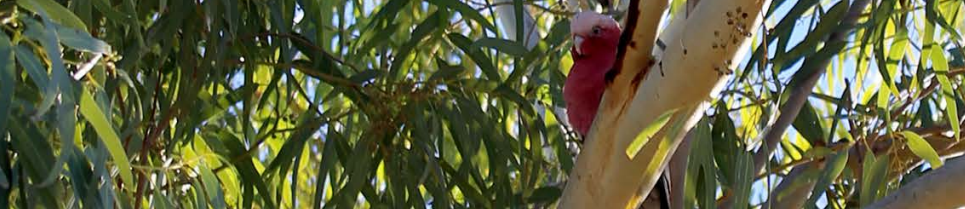

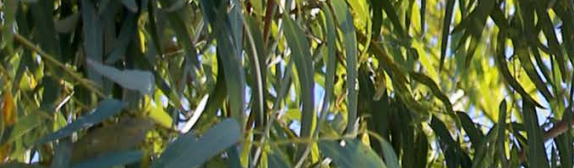

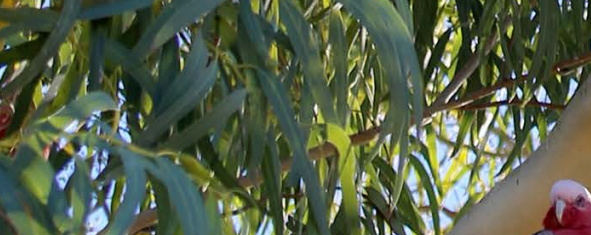

A.

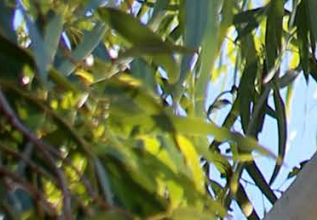

210

is 3 in

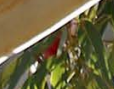

Q.2)

210. 50

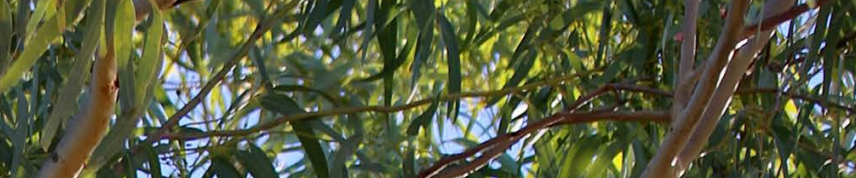

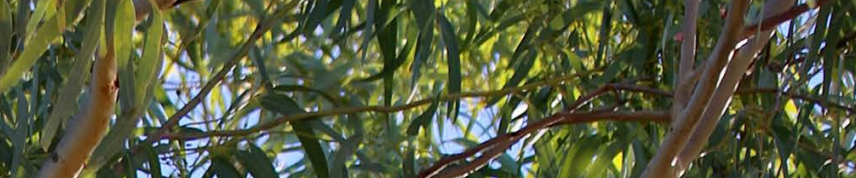

Tis

(1) 19

(1)

D. 2111

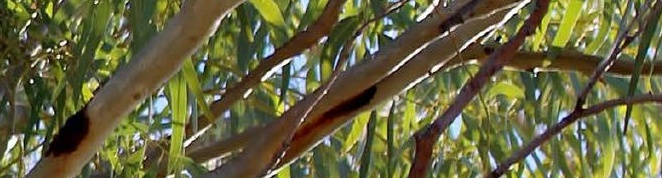

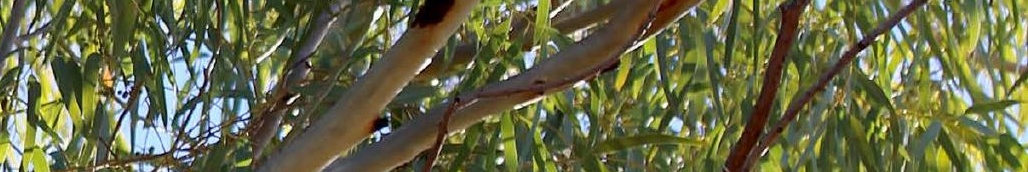
00
(4)
T.7.

1. 12

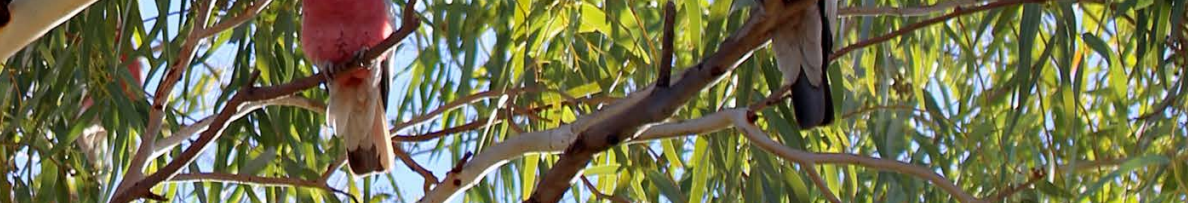

cons

. 

Le paragraphe 3.1 des lignes directrices de l'UICN pour la prise en charge d'organismes vivants confisqués explique l'utilité d'un un réseau consultatif sur la confiscation, qui peut fournir une orientation sur les soins immédiats à apporter à court terme aux individus confisqués.

Le mandat d'un tel groupe ou d'une telle équipe doit donc correspondre aux compétences, aptitudes et contacts nécessaires pour que les autorités puissent apporter, dans les plus brefs délais, des soins de court terme adaptés aux organismes vivants confisqués. Cela est nécessaire pour ne pas mettre en danger le bien-être des animaux ou la viabilité des animaux, des plantes et des champignons macroscopiques. Si les mesures prises à court terme réussissent, des options de placement à long terme des individus peuvent alors être envisagées.

Pour répondre à ce besoin, la composition du groupe ou de l'équipe doit être étudiée avec soin pour garantir au minimum l'accès aux connaissances nécessaires dans les domaines suivants:

- taxonomie, pour l'identification rapide et exacte des espèces/sous-espèces/variétés;

- médecine humaine et vétérinaire, pour la santé des êtres humains et des animaux concernés, y compris pour la mise en quarantaine;

- éthologie et élevage selon l'espèce;

- botanique selon l'espèce, dans les cas des plantes;

- droit;

- logistique, pour orienter le stockage et le transport.

Le groupe doit créer et maintenir des points de contact avec:

- aux niveaux local, régional et international, les centres de sauvetage/ réadaptation des animaux, les sanctuaires pour animaux, les zoos, et les jardins botaniques, ainsi que les associations correspondantes, qui sont susceptibles de fournir des conseils d'experts et, dans certains cas, d'héberger les organismes à court terme;

- le bureau national de l'Organisation mondiale de la santé animale (OIE) et les départements de médecine vétérinaire des gouvernements/universités, qui sont susceptibles de fournir des conseils sur la santé et le bien-être des animaux (www.oie.int);

- l'organe de gestion et l'autorité scientifique de la CITES au niveau national, ainsi que le secrétariat CITES (www.cites.org);

- les autorités policières et frontalières chargées de lutter contre les crimes sur la faune et la flore;

- d'autres instances et organismes consultatifs chargés de la santé et du 
bien-être des animaux, et des soins phytosanitaires, le cas échéant.

- les points de contact nationaux de la Convention internationale pour la protection des végétaux (CIPV), qui peuvent fournir une orientation sur les questions phytosanitaires (https://www.ippc.int/en/).

Le groupe doit nommer un ou plusieurs points de contact accessibles 24 heure sur 24 et les mettre à la disposition des autorités compétentes susceptibles de participer aux opérations de confiscation.

Le groupe ou l'équipe doit chercher à mettre en place des programmes de formation pour les autorités afin de les sensibiliser aux lignes directrices de I'UICN sur la prise en charge des organismes vivants confisqués, ainsi qu'aux directives applicables de la CITES, de I'UICN, de I'OIE, de la CIPV et de WAZA, entre autres.

Le groupe ou l'équipe doit chercher à nouer des liens avec les groupes ou équipes d'autres pays pour faciliter l'échange d'informations et de bonnes pratiques.

Les critères de sélection et le mandat du groupe ou de l'équipe devront peut-être être définis pour une région ou un pays particulier. Pour leur préparation, le réseau consultatif souhaitera peut-être se reporter à d'autres mandats existants, dont notamment:

- Ie mandat du groupe de spécialistes et du groupe de travail de la Commission de sauvegarde des espèces de l'UICN.

D'autres organismes et sources de référence importants sont:

- les normes d'excellence de la Global Federation of Animal Sanctuaries;

- I'European Association of Rescue Centres and Sanctuaries;

- I'Animal Welfare Strategy de WAZA;

- la résolution Conf. 17.8 de la CITES sur l'utilisation des spécimens d'espèces inscrites aux annexes de la CITES commercialisés illégalement et confisqués. 
Annexe 2 Sources d'informations utiles et liens hypertexte correspondants 

Directives AMVA sur l'euthanasie des animaux

https://www.avma.org/KB/Policies/Pages/Euthanasia-Guidelines.aspx

Résolution CITES Conf. 17.8 sur la disposition des spécimens d'espèces inscrites à la CITES commercialement illicites et confisqués

https://cites.org/eng/res/17/17-08.php

S'engager pour la conservation: la stratégie de conservation des zoos et aquariums du monde

https://www.waza.org/priorities/conservation/conservation-strategies/

Base de données mondiale de l'UICN sur les espèces envahissantes

http://www.iucngisd.org/gisd/

IUCN Species Survival Commission guidelines on the use of ex situ management for species conservation (Lignes directrices de l'UICN sur la gestion ex situ pour la conservation des espèces)

https://portals.iucn.org/library/node/44952

Lignes directrices de l'UICN Réintroductions et autres transferts de conservation

https://portals.iucn.org/library/node/45128

Liste rouge des espèces menacées de l'UICN

http://iucnredlist.org/

Groupes de spécialistes de la Commission de la survie des espèces de I'UICN

https://www.iucn.org/theme/species/about/species-survival-commission/ssc-specialist-group-directory

OIE-IUCN-EAZWV Quarantine and Health Screening Protocols for Wildlife prior to Translocation and Release into the Wild (Protocoles OIE-UICNEAZWV de mise en quarantaine et de contrôle sanitaire de la faune sauvage préalablement à la translocation et à la libération dans la nature)

https://portals.iucn.org/library/node/7971

Guidelines for wildlife disease risk analysis (Lignes directrices OIE-UICN pour l'analyse du risque de maladie chez les espèces sauvages)

https://portals.iucn.org/library/node/43385

Manual of procedures for wildlife disease risk analysis (Manuel de procédures de l'OIE-UICN pour l'analyse du risque de maladie chez les espèces sauvages)

https://portals.iucn.org/library/node/43386

Code sanitaire pour les animaux terrestres de l'OIE

http://www.oie.int/international-standard-setting/terrestrial-code/

Stratégie de WAZA sur le bien-être animal

https://www.waza.org/priorities/animal-welfare/animal-welfare-strategy/ 


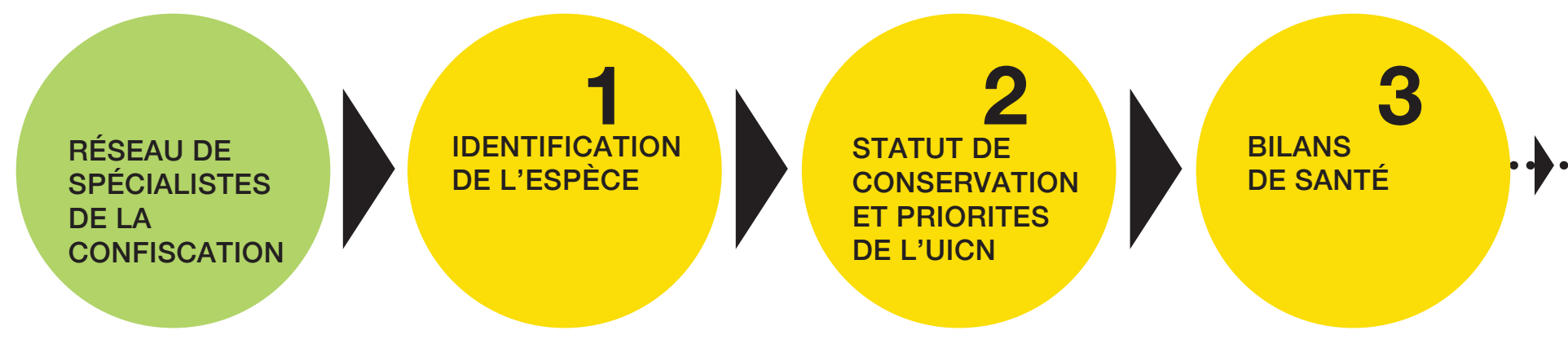

À quelle espèce appartient chaque individu confisqué ?

Un spécialiste est-il nécessaire à son identification (contacter un organisme de taxonomie)?

\begin{tabular}{l}
\hline Quelle est son aire de \\
répartition? \\
\hline L'espèce existe-t-elle dans \\
le pays de confiscation? \\
\hline Le pays de confiscation \\
appartient-il à l'aire de \\
répartition de l'espèce? \\
\hline Les autorités compétentes \\
ont-elles toutes été \\
consultées (par exemple en \\
ce qui concerne la \\
quarantaine)?
\end{tabular}

Un plan d'action pour la conservation de l'espèce a-t-il été mis en place? \begin{tabular}{l}
\hline L'espèce est-elle « menacée \\
" selon la Liste rouge des \\
espèces menacées de l'UICN \\
: est-elle considérée comme \\
"vulnérable ", "en danger " \\
ou " en danger critique "? \\
Voir le site www.redlist.org
\end{tabular}

Quelles sont les mesures de conservation de l'espèce recommandées par la Liste rouge de I'UICN?

Si l'espèce ne figure pas sur la Liste rouge de l'UICN ou si elle appartient aux catégories NE ("non-évaluée ») ou DD (« données insuffisantes »), demander conseil à la Commission de la sauvegarde des espèces de l'UICN.

L'individu appartient-il à une espèce dont la conservation doit faire l'objet d'une attention particulière au niveau national d'après les données de la Liste rouge du pays ou d'autres listes nationales de conservation?
Un bilan de santé conforme aux lignes directrices de I'UICN et de I'OIE sur l'appréciation des risques pour les maladies de la faune sauvage a-t-il été effectué ?

L'animal est-il « en forme »? Un individu est considéré comme "en forme "s'il a subi un examen vétérinaire complet ainsi qu'une période de quarantaine et s'il ne présente aucun signe de maladie infectieuse ou d'infirmité qui pourrait l'empêcher de survivre de façon autonome.

Si l'individu n'est pas " en forme ", un traitement pourrait-il remédier à la situation ? Dispose-t-on des installations et des ressources nécessaires au traitement requis?

Une euthanasie immédiate est-elle nécessaire pour éviter à l'animal de souffrir plus longtemps? Cette décision nécessite une évaluation réalisée par un vétérinaire ou un expert. 


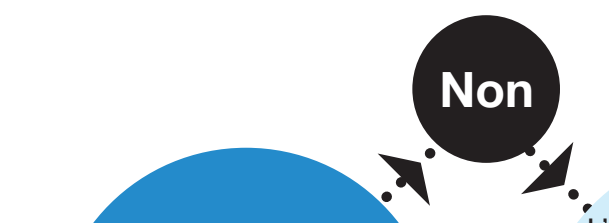

L'animal

L'individu peut-il retrouver suffisamment la santé et une bonne forme physique pour (1) avoir une chance raisonnable de survivre dans la nature et (2) ne est-il en forme?

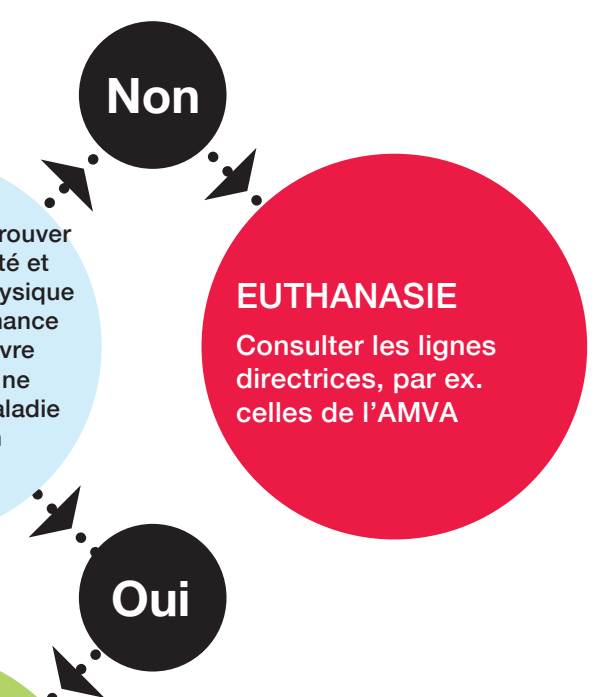

Transfert vers un centre de détention immédiate disposant d'installations appropriées pour assurer le bien-être et la survie de l'individu
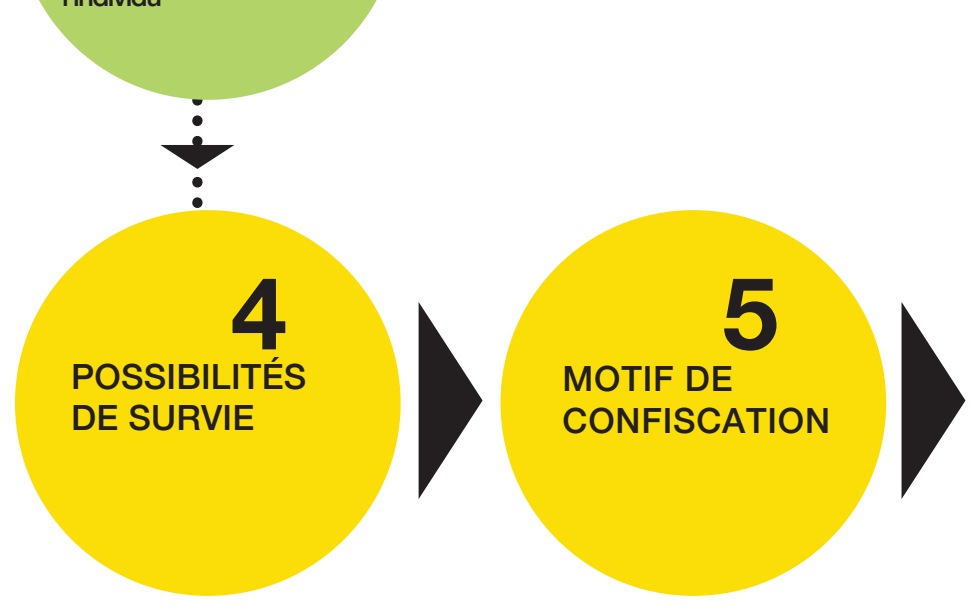

L'individu a-t-il été retiré de

L'individu appartient-il à une son habitat naturel ?

S'agit-il d'un individu né en captivité ou ayant fait l'objet d'une reproduction artificielle?

L'espèce est-elle concernée par un programme d'élevage de conservation?

Si oui, l'individu peut-il participer à un programme de ce type?

\section{espèce pouvant être}

commercialisée légalement?

Si oui, les procédures appropriées applicables à son commerce ou à sa possession ont-elles été respectées?

Est-il probable que les procédures et démarches administratives appropriées seront réalisées dans un avenir proche?

Si oui, quand?

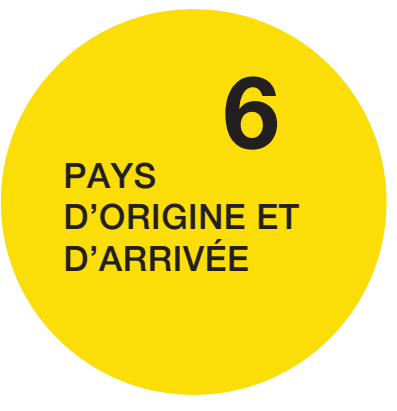

Où l'individu confisqué a-t-il été capturé : dans quel pays, dans quelle région?

Si cette information n'est pas disponible, l'endroit peut-il être identifié ?

Le pays d'origine est-il signataire de la Convention sur la diversité biologique, du Protocole de Nagoya sur l'accès et le partage des avantages (APA), de la Convention sur le commerce international des espèces menacées d'extinction (CITES) ou du Traité international sur les ressources phytogénétiques pour l'alimentation et l'agriculture (ITPGRFA) ?

Le pays d'origine de l'individu est-il le même que celui d'arrivée ?

Le pays d'arrivée est-il prêt à s'occuper de l'individu confisqué ?

Le rapatriement pose-t-il certains problèmes quant au bien-être de l'individu? 

Collecte d'i

Identification de l'espèce

Bilans de santé Statut de conservation et priorités de
I'UICN

Possibilités

L'espèce a-t-elle de la valeur du poin de la conservation?

Non : attention à l'allocation des ressources lorsqu'elles sont limitées

Peut-il être rapatri

Mise en quarantaine/détention

jusqu'à l'achèvement des

démarches administratives

\section{RAPATRIEMENT}

Retour dans le pays d'origine, au sein d'une organisation autorisée et/ou d'installations " agréées ", c'est-à-dire celles créées pour gérer les animaux confisqués au titre de la CITES

Suivre les Lignes directrices de prise en charge des organismes vivants confisqués (ce document)

\section{TRANSFERT}

Se référer aux Lignes directr réintroductions et les autres la sauvegarde
MISE A MORT SANS

CRUAUTE/DESTRUCTION

Consulter les lignes directrices, par ex. celles de I'AMVA 



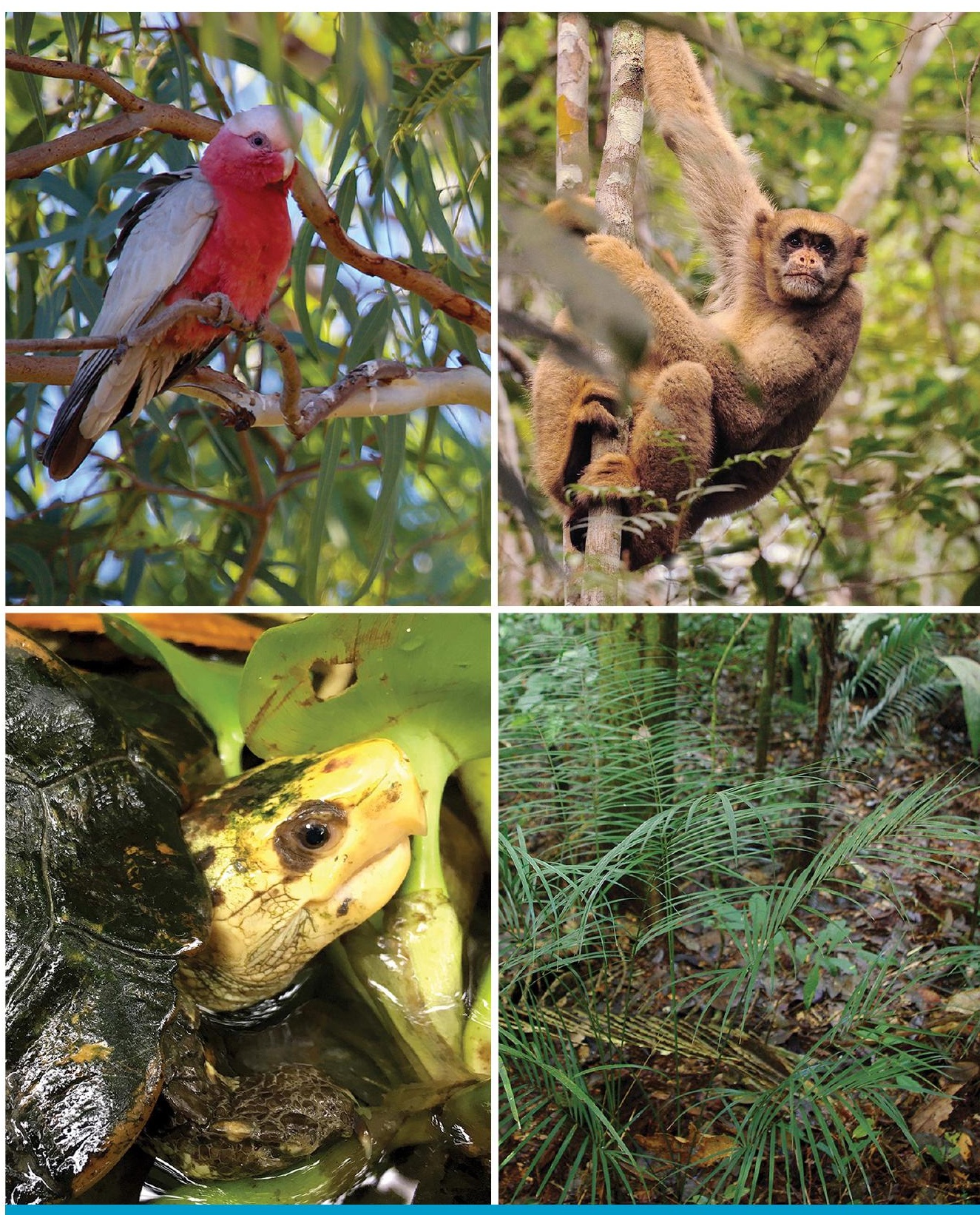

UNION INTERNATIONALE

POUR LA CONSERVATION DE LA NATURE

SIĖGE MONDIAL

Rue Mauverney 28

1196 Gland, Suisse

mail@iucn.org

Tél. +41229990000

Fax +41 229990002

www.iucn.org/fr 